\title{
openheart Revascularisation of left main stem disease: a prospective analysis of modern practice and outcomes in a non- surgical centre
}

\author{
J J Coughlan, ${ }^{1}$ Nial Blake, ${ }^{1}$ Napohn Chongprasertpon, ${ }^{1}$ Munir Ibrahim, ${ }^{1}$ \\ Samer Arnous, ${ }^{2}$ Thomas John Kiernan ${ }^{1}$
}

\begin{abstract}
- Additional material is published online only. To view please visit the journal online (http://dx.doi.org/10.1136/ openhrt-2018-000804).
\end{abstract}

To cite: Coughlan JJ, Blake N, Chongprasertpon N, et al. Revascularisation of left main stem disease: a prospective analysis of modern practice and outcomes in a non-surgical centre. Open Heart 2018;5:e000804. doi:10.1136/ openhrt-2018-000804

Received 16 February 2018 Revised 7 May 2018 Accepted 13 June 2018

Check for updates

\section{(c) Author(s) (or their} employer(s)) 2018. Re-use permitted under CC BY-NC. No commercial re-use. See rights and permissions. Published by BMJ.

${ }^{1}$ Department of Cardiology, University Hospital Limerick, Dooradoyle, Ireland 2Department of Cardiology, Rigshospitalet, Copenhagen, Denmark

Correspondence to Dr J J Coughlan; jjcoughı@ gmail.com

\section{ABSTRACT}

Purpose In this study, we sought to prospectively analyse the management and long term outcomes associated with revascularisation of left main stem disease via percutaneous coronary intervention $(\mathrm{PCl})$ and coronary artery bypass grafting (CABG) in our centre.

Methods This prospective study enrolled all patients with unprotected left main stem disease undergoing revascularisation from January 2013 to June 2014. Baseline characteristics, hospital presentation and hospital stay length were collected. Patients were followed up at 1, 2 and 3 years. Primary outcomes of Major Adverse Cardiovascular and Cerebrovascular Events (MACCE) were defined as death, $Q$ wave myocardial infarction, stroke, repeat revascularisation and readmission within 30 days.

Results 56 patients with significant left main stem coronary artery disease were identified from the clinical registry. 27 patients underwent $\mathrm{PCI}$ (median age 67.7) and 29 CABG (median age 68.6). PCI patients had a higher surgical risk as measured by mean euroSCORE $(4.95 \pm 5.8$ vs $3.11 \pm 3.85)$. At 3 years, total MACCE occurred in $29.6 \%$ of the $\mathrm{PCl}$ cohort and $27.5 \%$ of the CABG cohort. Death occurred in three patients in the $\mathrm{PCI}$ group within the first 6 months. Death occurred in one patient in the CABG group over 2 years postprocedure. Two patients in the CABG cohort presented with Transient Ischemic Attacks (TIAs) at 2-year follow-up. At 3 years, revascularisation occurred in three patients in the $\mathrm{PCl}$ cohort. There were no revascularisation events in the $\mathrm{CABG}$ cohort.

Conclusions $\mathrm{PCl}$ with modern drug eluting stents is a reasonable treatment option for unprotected left main stem disease in a non surgical centre.

\section{INTRODUCTION}

Coronary artery disease (CAD) remains the leading cause of death worldwide. ${ }^{12}$ The left ventricle is responsible for the generation of cardiac output and primarily depends on the left main coronary artery (LMCA) for its blood supply. ${ }^{3}$ Compromise of this vessel has catastrophic outcomes for many individuals. Significant LMCA disease refers to stenosis of the luminal diameter of greater than $50 \%$ and is observed in $4.8 \%$ of patients undergoing cardiac angiography. ${ }^{4}$ Patients

\section{Key questions}

What is already known about this subject?

- Coronary artery bypass grafting remains the gold standard revascularisation treatment option for the revascularisation of left main stem disease. However, as interventional techniques have evolved, percutaneous coronary intervention (PCI) has become an increasingly used treatment modality.

What does this study add?

- This study prospectively analyses the management and outcomes of left main stem disease in a non-surgical centre. This provides insight into real world decision making and patient outcomes with regard to left main stem revascularisation in modern clinical practice.

How might this impact on clinical practice?

- Our study provides evidence that $\mathrm{PCl}$ with modern drug eluting stents is a reasonable treatment option for unprotected left main stem disease in a non-surgical centre, particularly in emergent cases presenting with ST elevation Myocardial Infarction (STEMI)/ Cardiogenic shock and in patients deemed to be at too high a risk for surgical revascularisation after discussion by the HEART team.

with LMCA disease have a mortality rate of approximately $50 \%$ at 5 years with medical management. ${ }^{5}{ }^{6}$ The LMCA is defined as 'Protected' when there is at least one patent bypass graft to the left circumflex or left anterior descending artery. In the absence of such a patent graft, the LMCA is said to be unprotected.

Percutaneous coronary intervention (PCI) and coronary artery bypass grafting (CABG) are the primary treatment options for coronary revascularisation in patients with stable CAD and ischaemia. ${ }^{7}$ CABG remains the gold standard in the treatment of LMCA disease and has a long history of both safety and efficiency. ${ }^{89}$ However, as interventional 
techniques have developed, the outcomes of patients with left main disease treated with PCI have improved. This has served to threaten the long-standing position of CABG and PCI is now considered a viable treatment alternative for unprotected LMCA disease in some cases. ${ }^{10}$

Decision making regarding the use of PCI versus CABG in unprotected left main stem coronary artery disease (LMCAD) continues to be an area of ongoing debate. According to the recent American and European revascularisation guidelines, a physician's decision to opt for PCI or CABG is dependent on several factors. These include the patient's own preference, comorbidities and the complexity of their CAD. ${ }^{89}$ There is broad consensus that uncomplicated single or double vessel CAD is amenable to PCI, whereas in more complex three vessel CAD a CABG is warranted. ${ }^{8}{ }^{9}$ However, factors such as lesion location, impact on blood flow, degree of stenosis and calcification also play a key role in clinical decision making. ${ }^{7}$ In order to formulate the optimal revascularisation approach, patients should be provided with the relevant information to make an informed choice. As per the ESC guidelines, the most valuable advice will be provided to them by the Heart Team. ${ }^{9}$

Since July 2013, PCI for unprotected LMCA stenosis has been performed in the cardiology department of the University Hospital Limerick (UHL). The primary aim of this study was to assess the outcomes of patients with unprotected significant LMCA stenosis $(>50 \%)$ who underwent PCI in the cardiology department of UHL in 2013 and 2014 and compare this to the patients who underwent CABG for unprotected left main disease within the same time frame.

\section{METHODS}

This prospective observational study was vetted and approved by the UHL research and ethics committee. We identified both PCI and CABG patients at baseline with unprotected Left Main Stem (LMS) disease requiring intervention. Patients with significant ULMCA disease who attended UHL between January 2013 and July 2014, and subsequently underwent PCI or CABG, were identified from the clinical registry. Consent to a telephone interview was obtained for follow-up data collection in the occurrence of MACCE and quality of life (QoL) questionnaires. Inclusion criteria were patients with unprotected LMS disease who were male or female within the time frame of January 2013 to June 2014. The only exclusion criterion was protected LMS disease.

Patients undergoing PCI had their intervention in UHL. As UHL is a non-surgical centre, CABG patients were referred to cardiothoracic facilities in Dublin and Cork. Baseline characteristics, hospital presentation and hospital stay length were collected via detailed chart analysis. SYNTAX score was calculated for each subject using coronary angiography imaging software by a consultant cardiologist. EuroSCORE was also calculated for all patients. Patient records were accessed via chart analysis initially and via McKesson radiology software to follow patients for MACCE and readmissions. Patient electronic records were accessed via McKesson cardiology software to follow for revascularisation events. Patients were followed up via telephone interview at 1, 2 and 3 years over a period of 1 week only. Primary outcomes of MACCE were defined as death, $Q$ wave myocardial infarction, stroke, repeat revascularisation and readmission within 30 days. QOL was determined via angina severity (CCS score), heart failure status (NYHA classification) and the quality of life (EQD5L) tool. Secondary outcomes of MACCE at 2 and 3 years were defined as death, recurrent MI, stroke/TIA and the need for repeat revascularisation via $\mathrm{CABG}$ or alternatively PCI.

Analysis was performed using SPSS for MAC OS. Categorical variables were compared across both groups using Fischer's exact test or the $\chi^{2}$ test where appropriate. Continuous variables were compared using the MannWhitney test and the Student's t-test as appropriate. A p value of $<0.05$ was considered statistically significant.

\section{RESULTS}

\section{Baseline demographics}

Fifty-six patients with significant LMCAD attending UHL were identified from the clinical registry. Twenty-seven patients underwent PCI and 29 CABG (table 1). Mean age was $69 \pm 10.4$ years in the PCI group and $65.1 \pm 15.3$ years in the CABG group $(\mathrm{p}=0.71)$. The majority of patients were male ( $85 \%$ of PCI cohort, $82 \%$ of the CABG group). A history of myocardial infarction at presentation was also common to both cohorts (PCI $51.9 \%$ vs $41.4 \%$, $\mathrm{p}=0.59$ ). There were similar levels of angina pectoris in both groups. Diabetes was present in $18.5 \%$ of the PCI cohort and $17.2 \%$ of the CABG group. Of note, there was a higher prevalence of renal impairment in the CABG cohort $(\mathrm{p}<0.01)$. PCI patients had a higher surgical risk as measured by mean euroSCORE $(4.95 \pm 5.8$ vs $3.11 \pm 3.85)$. Of note, more patients in the PCI group were classed as high surgical risk (euroSCORE $>6$ ) compared with the CABG group (19.2\% vs $6.9 \%, \mathrm{p}=0.17)$. Mean SYNTAX score was $33.5 \pm 17.6$ in the PCI group and $48.4 \pm 11.7$ in the CABG cohort. The majority of patients in the PCI cohort had a SYNTAX score of 0-32 (70.4\%), while the majority of patients undergoing CABG had a SYNTAX score of $>33(93.1 \%)$. The PCI group had a shorter length of stay (6.6 vs 37.6 days, $\mathrm{p}=0.06$ ) and patients allocated to $\mathrm{CABG}$ had to wait longer for their revascularisation (PCI 3.4 vs $\mathrm{CABG} 8$ days, $\mathrm{p}=0.04$ ).

\section{Presentation}

It is important when considering differences in MACCEs between the two groups to remember that the pattern of presentation between the two groups was different. Thirty-seven per cent of the LMS PCI procedures were performed as salvage procedures in the setting of STEMI \pm cardiogenicshock whereas none of the CABG procedures were performed as salvage procedures $(37 \%$ 
vs $0 \%, \mathrm{p}<0.005)$ with all patients being classed as either elective $(27 \%)$ or urgent, that is, performed as an inpatient $(73 \%)$. Also, as mentioned above, these patients were more likely to be high surgical risk (euroSCORE $>6$ ) although their SYNTAX score tended to be lower.

\section{Procedural characteristics}

For patients undergoing PCI to the LMS, we also analysed the procedural characteristics. PCI was performed in 27 patients with an average of $1.44 \pm 0.64$ stents deployed per patient. Mean diameter of stent deployed was $3.48 \pm 0.29 \mathrm{~mm}$. Bifurcation stenting was performed in $18.5 \%$ of cases. Drug eluting stents were used in all cases. In $81.5 \%$ of cases, everolimus eluting stents were used. Intracoronary imaging was used in $48.15 \%$ of cases. Intravascular ultrasound (IVUS) was used in $61.5 \%$ of these cases with the remainder using optical coherence tomography (OCT). $14.8 \%$ of patients presented in cardiogenic shock and mechanical devices were used in $18.5 \%$ of cases. The most common mechanical device used was intra-aortic balloon pump which was used in four cases. The Impella device was used in the remaining case. One patient required a temporary pacing wire and two patients required emergency intubation, both in the setting of STEMI and cardiogenic shock. Rotational atherectomy was used in one case to facilitate stent delivery in a heavily calcified vessel.

\section{Primary endpoint}

The primary endpoint for this study was MACCEs at 1 , 2 and 3 years. This was a composite endpoint of death, stroke/TIA, $Q$ wave myocardial infarction, repeat revascularisation and readmission within 30 days. Given the patient numbers in this trial, it is possible for us to look into the primary endpoints in detail. We detail these below.

\section{1 year MACCE}

\section{Mortality}

There were three deaths in the PCI cohort within the first 6 months postintervention. One elderly patient $(>90$ years) represented with Acute Coronary Syndrome (ACS) and died during inpatient stay. Another patient died of colorectal cancer. There were no deaths at 1 year in the CABG group (online supplementary table 2).

\section{Stroke/TIA}

There were no strokes/TIAs in either group at 1 year.

\section{Myocardial infarction/Repeat revascularisation}

One patient presented with myocardial infarction requiring repeat revascularisation and subsequently died. There were no myocardial infarctions or repeat revascularisations in the CABG cohort.

\section{0-day readmission}

There was one readmission with acute coronary syndrome in the PCI group. In the CABG group, there were five 30 -day readmissions $(17.2 \%)$. These consisted of the following reasons. Two patients were readmitted complaining of pleuritic chest pain. One patient had a syncopal episode. Two patients were readmitted with postCABG sternal wound infections with one patient having a CT thorax confirming osteomyelitis of the sternum requiring prolonged antibiotic therapy.

\section{MACCES}

Total MACCEs at 1 year in the two groups were $22.2 \%$ in the PCI cohort and $17.2 \%$ in the CABG cohort $(\mathrm{p}=0.64)$.

\section{2 years MACCE}

Mortality

There were no further deaths in the PCI cohort. There were also no deaths in the CABG cohort at 2-year follow-up.

\section{Stroke/TIA}

Two patients in the CABG cohort presented with TIAs at 2-year follow-up. Of the PCI cohort, MACCE of death of any cause was recorded in three patients.

\section{Myocardial infarction/Repeat revascularisation}

One patient in the PCI cohort was admitted with Myocardial Infarction (MI) and consequently this patient was referred for CABG for revascularisation. There were no episodes of MI or repeat revascularisation in the CABG cohort.

\section{MACCES}

There were no differences in cumulative MACCE between the groups at 2 years (22.2\% vs $24.1 \%, \mathrm{p}=0.86$ ) (online supplementary table 3 ).

\section{3 years MACCE \\ Mortality}

There were no further deaths in the PCI cohort. One patient passed away in the CABG cohort. Mortality total was 3 in the PCI cohort and 1 in the CABG cohort $(11.1 \%$ vs $3.4 \%, p=0.27$ ) (online supplementary table 4 ). Cardiac mortality at 3 years was $3.7 \%$ in the PCI cohort and $3.4 \%$ in the CABG cohort $(\mathrm{p}=0.99)$.

\section{Stroke/TIA}

There were no further strokes/TIAs in either the PCI or CABG cohort at 3 year follow-up. The occurrence of stroke or TIA at 3 years was $0 \%$ in the PCI group and $6.9 \%$ in the $\mathrm{CABG}$ group $(0 \%$ vs $6.9 \%, \mathrm{p}=0.17)$

\section{Myocardial infarction/Repeat revascularisation}

In the PCI cohort, there were two further revascularisation events at 3-year follow-up. One patient presented with a STEMI requiring PPCI and one patient had a further elective PCI performed. There were no revascularisation procedures carried out in the CABG cohort. There were a total of two myocardial infarctions and three revascularisation events in the PCI group and no myocardial infarction or repeat revascularisation in the CABG group at 3 years (myocardial infarction: $7.4 \%$ vs $0 \%, \mathrm{p}=0.14$; revascularisation: $11.1 \%$ vs $0 \%, \mathrm{p}=0.07$ ). 


\section{MACCEs}

There was no statistical difference in major MACCE between the groups at 3 years $(29.6 \%$ vs $27.5 \%, \mathrm{p}=0.99)$. However, if we exclude 30-day readmission from the analysis, the difference in MACCE between the groups nearly reaches statistical significance with an excess of MACCE in the PCI cohort (29.6 vs $10.3 \%, \mathrm{p}=0.07$ ).

\section{QUALITY OF LIFE}

The PCI and CABG groups did not differ in terms of QoL with short-term follow-up at 1 year. However, from a qualitative point of view, these data are interesting and inform us of the patient's own perspective on their health. The psychological comorbidity of cardiac patients is highlighted by this questionnaire where $22.7 \%$ and $25.9 \%$ of PCI and CABG-treated patients described themselves as moderately anxious or depressed. Mean EQ5D thermometer scores were broadly similar between groups $(72.14 \% \pm 20.22 \%$ vs $73.86 \pm 17.92 \%, p=0.77)$ at 3 years. The EQ5D score is based on a scale in which the best health state the patient can imagine is marked by 100 and the worst health state by 0 .

NYHA classification and CCS angina severity scale scores at 3 years were also calculated for all patients and found to be similar between PCI and CABG cohorts (NYHA Class: $1.8 \pm 0.93$ vs $1.58 \pm 0.67, \mathrm{p}=0.38$, CCS class: $1.50 \pm 0.98$ vs $1.6 \pm 0.67, \mathrm{p}=0.70)$. This suggests that the symptomatic response to revascularisation is equal in both groups.

\section{SERVICE PROVISION}

The PCI group had a shorter length of stay (6.6 vs 37.6 days, $\mathrm{p}=0.06$ ). UHL is a non-surgical centre and patients needed to be transferred to other hospitals in order to undergo CABG. Unsurprisingly, as a result patients allocated to $\mathrm{CABG}$ had to wait significantly longer for their revascularisation ( 3.4 vs 8 days, $\mathrm{p}=0.04$ ).

\section{DISCUSSION}

Decision making with regard to revascularisation of LMCA disease is undoubtedly complex and influenced by a variety of factors including patient characteristics, coronary artery anatomy and clinical presentation. Guidelines exist in order to help professionals deliver optimal management for their patients. However, the final decision will likely be determined by the healthcare professional based on best evidence, after consultation with the patient, taking his/her preferences and individual circumstances into account. We will now discuss some aspects of our study with regard to decision making regarding revascularisation and outcomes after the same.

ESC Guidelines ${ }^{9}$ specify certain recommendations with regard to revascularisation of left main disease in patients with SCAD and low surgical risk based on SYNTAX score. For patients with LMCA disease and a syntax score of $<22$, CABG and PCI both receive class IB recommendations. For patients with LMCA disease and a SYNTAX score of
22-32, CABG receives a class IB recommendation but PCI receives a class IIa $\mathrm{B}$ recommendation. For patients with LMCA disease and a SYNTAX score of $>32$, CABG receives a IB recommendation and PCI receives a class IIIB recommendation. Looking at our cohort, we managed our patients broadly in line with the criteria for patients with a SYNTAX score of $<22$ and those with a SYNTAX score of $>32$. Those with a score of $<22$ received PCI in $100 \%$ of cases $(100 \%$ vs $0 \%, \mathrm{p}=<0.001)$. Those with a SYNTAX score of $>32$ were managed predominantly with CABG $(77.1 \%$ vs $22.9 \%, \mathrm{p}=<0.001)$. However, in patients with an intermediate SYNTAX score (22-32), the predominant method of revascularisation was PCI $(88.2 \%$ vs $11.8 \%$, $\mathrm{p}=<0.001)$. This goes against ESC guidelines which tend to favour $\mathrm{CABG}$ in this group of patients.

As mentioned in our results, a significant number of patients revascularised with PCI had their procedure performed as a 'salvage' (37\%) or urgent case (33\%). As UHL is a non-surgical centre, emergency CABG is not able to be performed on site and consequently, $0 \%$ of CABG procedures were deemed 'salvage' cases. A significant proportion of these patients presented in cardiogenic shock $(14.8 \%)$. Some of these patients were also deemed unsuitable for CABG and it should be noted that they had a numerically higher mean euroSCORE and a higher proportion of patients with euroSCORE $>6$, marking them as high surgical risk. As such, our PCI patients may represent a more clinically unwell cohort of patients. The patients in the CABG group tended to have higher SYNTAX Scores (48.4 vs 33.5, $\mathrm{p}=0.24$ ) which has been shown to confer higher risk of adverse outcomes with PCI but not with CABG. Salvage CABG is a relatively rare procedure in modern PCI. Haan et al. reported that from 1994 to 2003, the rate of salvage CABG decreased from $2.9 \%$ to $0.8 \%$ of all procedures. ${ }^{11}$ This was defined as CABG performed within 6 hours of PCI.

Given the small number of patients in our study, it has been possible for us to forensically analyse the events that occurred in both groups. With regard to mortality, there was only one death in the CABG group at 3-year follow-up. While the mortality rate in the PCI group was higher at 3 years $(11.1 \%$ vs $3.7 \%, \mathrm{p}=0.27)$, one of these events was from an unrelated illness (colorectal cancer). Obviously these patients would never have been surgical candidates based on their comorbidities. The cardiac mortality at 3 years was similar between the two groups $(3.4 \%$ vs $3.7 \%$, $\mathrm{p}=0.99$ ). Care must be taken when comparing our results to international data due to the inherent differences in patient populations being studied. A study from Italy ${ }^{12}$ analysed long-term outcomes in patients unsuitable for surgical revascularisation, undergoing elective LMCA angioplasty. They reported an all-cause mortality rate of $14 \%$ with a mean follow-up time of $14 \pm 10.8$ months. In an American study of patients with increased surgical risk (Mean euroSCORE $5.2 \pm 0.4,41 \%$ euroSCORE $>6$ ), cardiac mortality was $12 \%$ and all-cause mortality $21 \%$, with a mean follow-up of $28 \pm 1$ months ${ }^{13}$. Similarly, the LEFT-MAIN ISAR study reported 2-year mortality of 
$10.7 \%$ in the paclitaxel eluting stent group and $8.7 \%$ in the sirolimus eluting stent group. ${ }^{14}$ Emergent LMS PCI has been reported to have less favourable outcomes. The BCIS national audit compared outcomes between occlusive and non-occlusive LMS disease. ${ }^{15}$ Mortality rates were high in both groups at 3 years $(73.9 \%$ vs $52.3 \%, \mathrm{p}<0.001)$, though significantly higher in the LMS occlusion group. While these studies vary with respect to patient population, presentation (elective vs emergency) and follow-up, they provide a reference range for our results, which appear to be broadly in keeping with the international literature. Unfortunately, there is no published data regarding outcomes for left main stem revascularisation in Ireland. We feel that our heterogenous patient population, consisting of both emergency left main revascularisation and elective procedures post HEART team discussion, is likely reflective of real world practice.

TIA occurred in only two CABG patients. Interestingly, this occurred over 1-year post-CABG and not in the immediate postoperative period. There were no TIA/ Stroke episodes in the PCI group, despite this group having a higher proportion of patients with a history of cerebrovascular events $(14.8 \%$ vs $3.4 \%, \mathrm{p}=0.19)$. Revascularisation and MI were higher in the PCI group. While 30-day readmissions were more common in the CABG group, the majority of these were not serious, except for one case of sternal osteomyelitis requiring long-term antibiotic therapy.

A couple of meta-analyses by Sá et al suggested CABG was the best treatment for ULMCA disease with higher risk of target vessel revascularisation as well as MACCE in the PCI group. ${ }^{16}{ }^{17}$ Sá et al recently published another meta-analysis suggesting similar results but with no significant difference in MACCE at 1-year follow-up. ${ }^{18}$ The authors mentioned that no definitive conclusion can be made from the evidence due to heterogeneity in study outcomes, procedural strategies and complexity of coronary lesions. ${ }^{18}$ Unlike the previous meta-analyses, ${ }^{16}{ }^{17}$ the more recent one ${ }^{18}$ found no significant difference in MACCE which may represent improvement of outcomes associated with the evolution of interventional techniques.

Our study prospectively analyses the management of and outcomes associated with left main stem revascularisation in our centre. It provides an insight into the real world management of this challenging condition beyond the clinical guidelines which are well recognised. Reassuringly, patients who undergo modern PCI and CABG both appear to have excellent outcomes at 3 years postprocedure. Patients undergoing PCI appear to have an increased need for revascularisation and there was an excess of cerebrovascular events in the CABG group. The excess mortality in the PCI cohort may reflect the patient comorbidities rather than the method of revascularisation.

Emergent LMCA revascularisation in a non-surgical centre remains a challenge, although of the patients undergoing PCI as salvage or urgent cases in our centre,
$90 \%$ survived to 3 years. This serves to highlight how far PCI has come since coronary angioplasty was introduced 40 years ago. This compares with the two major randomised trials on LMS angioplasty versus CABG, NOBLE and EXCEL. The NOBLE trial ${ }^{19}$ reported 5-year survival of $88 \%$ for PCI in treatment of unprotected LMS disease compared with $91 \%$ for CABG ( $p=0.77$ ) and the EXCEL trial ${ }^{20}$ reported $91.8 \%$ survival for PCI and $94.1 \%$ in the CABG cohort at 3 years. Overall, our study highlights the need for guideline-based individualised treatment planning for patients undergoing LMCA revascularisation. This decision making process should take multiple factors into account including clinical guidelines, patient preference, patient comorbidities and HEART team discussion. For patients requiring emergent LMS revascularisation in a non-surgical centre, it provides reassurance that this can be performed via PCI with short and long-term outcomes that are comparable to the published international literature.

\section{CONCLUSION}

PCI with modern drug eluting stents is a reasonable treatment option for unprotected left main stem disease in a non-surgical centre, particularly in emergent cases presenting with STEMI/Cardiogenic shock and in patients deemed to be at too high a risk for surgical revascularisation.

Contributors JJC was primarily responsible for drafting the manuscript. NB assisted with drafting of the manuscript, data collection and analysis and is co-first author. NC assisted with data collection, data analysis and manuscript revision. MI and SA provided editorial feedback for the manuscript. TJK was primarily responsible for the project concept and provided editorial feedback.

Funding The authors have not declared a specific grant for this research from any funding agency in the public, commercial or not-for-profit sectors.

Competing interests None declared.

Patient consent Not required.

Ethics approval University Hospital Limerick.

Provenance and peer review Not commissioned; externally peer reviewed.

Data sharing statement No additional data are available.

Open access This is an open access article distributed in accordance with the Creative Commons Attribution Non Commercial (CC BY-NC 4.0) license, which permits others to distribute, remix, adapt, build upon this work non-commercially, and license their derivative works on different terms, provided the original work is properly cited, appropriate credit is given, any changes made indicated, and the use is non-commercial. See:http://creativecommons.org/licenses/by-nc/4.0/.

\section{REFERENCES}

1. Mathers CD, Loncar D. Projections of global mortality and burden of disease from 2002 to 2030. PLoS Med 2006;3:e442.

2. Gaziano TA, Bitton A, Anand S, et al. Growing epidemic of coronary heart disease in low- and middle-income countries. Curr Probl Cardiol 2010;35:72-115.

3. Kalbfleisch $\mathrm{H}$, Hort W. Quantitative study on the size of coronary artery supplying areas postmortem. Am Heart J 1977;94:183-8.

4. Giannoglou GD, Antoniadis AP, Chatzizisis YS, et al. Prevalence of narrowing $>$ or $=50 \%$ of the left main coronary artery among 17,300 patients having coronary angiography. Am J Cardiol 2006;98:1202-5.

5. Cohen MV, Gorlin R. Main left coronary artery disease. Clinical experience from 1964-1974. Circulation 1975;52:275-85. 
6. Taylor HA, Deumite NJ, Chaitman BR, et al. Asymptomatic left main coronary artery disease in the Coronary Artery Surgery Study (CASS) registry. Circulation 1989;79:1171-9.

7. Head SJ, Farooq V, Serruys PW, et al. The SYNTAX score and its clinical implications. Heart 2014;100.

8. Windecker S, Kolh P, Alfonso F, et al. ESC/EACTS Guidelines on myocardial revascularization. European Heart Journal 2014;35:2541-619.

9. Hillis LD, Smith PK, Anderson JL, et al. 2011 ACCF/AHA Guideline for coronary artery bypass graft surgery. a report of the American College of Cardiology Foundation/American Heart Association Task Force on Practice Guidelines. Developed in collaboration with the American Association for thoracic surgery, society of cardiovascular anesthesiologists, and society of thoracic surgeons. J Am Coll Cardiol 2011;58:e123-e210.

10. Deb S, Wijeysundera HC, Ko DT, et al. Coronary artery bypass graft surgery vs percutaneous interventions in coronary revascularization: a systematic review. JAMA 2013;310:2086-95.

11. Haan CK, O'Brien S, Edwards FH, et al. Trends in emergency coronary artery bypass grafting after percutaneous coronary intervention, 1994-2003. Ann Thorac Surg 2006;81:1658-65.

12. Vignali L, Talanas G, Menozzi A, et al. Long-term mortality in patients unsuitable for surgical revascularization undergoing elective left main coronary artery angioplasty. Minerva Cardioangiol 2008;56:1-11.

13. Wood FO, Saylors EK, Schneider JE, et al. Unprotected left main disease managed with drug-eluting stents: long-term outcome of 100 patients with increased surgical risk. Catheter Cardiovasc Interv 2008;71:533-8.
14. Mehilli J, Kastrati A, Byrne RA, et al. Paclitaxel- versus sirolimuseluting stents for unprotected left main coronary artery disease. $J$ Am Coll Cardiol 2009;53:1760-8.

15. Patel N, De Maria GL, Kassimis G, et al. Outcomes after emergency percutaneous coronary intervention in patients with unprotected left main stem occlusion: the BCIS national audit of percutaneous coronary intervention 6-year experience. JACC Cardiovasc Interv 2014;7:969-80.

16. Sá MP, Ferraz PE, Escobar RR, et al. Five-year outcomes following $\mathrm{PCl}$ with DES versus CABG for unprotected LM coronary lesions: meta-analysis and meta-regression of 2914 patients. Rev Bras Cir Cardiovasc 2013;28:83-92.

17. Sá MP, Soares AM, Lustosa PC, et al. Meta-analysis of 5,674 patients treated with percutaneous coronary intervention and drug-eluting stents or coronary artery bypass graft surgery for unprotected left main coronary artery stenosis. Eur $J$ Cardiothorac Surg 2013:43:73-80.

18. Sá M, Soares AF, Miranda RGA, et al. CABG Surgery Remains the best Option for Patients with Left Main Coronary Disease in Comparison with PCI-DES: Meta-Analysis of Randomized Controlled Trials. Braz J Cardiovasc Surg 2017;32:408-16.

19. Mäkikallio T, Holm NR, Lindsay M, et al. Percutaneous coronary angioplasty versus coronary artery bypass grafting in treatment of unprotected left main stenosis (NOBLE): a prospective, randomised, open-label, non-inferiority trial. Lancet 2016;388:2743-52.

20. Stone GW, Sabik JF, Serruys PW, et al. Everolimus-eluting stents or bypass surgery for left main coronary artery disease. $N$ Engl J Med 2016;375:2223-35 